\title{
Improving solar wind persistence forecasts: removing transient space weather events, and using observations away from the Sun-Earth line
}

Article

Published Version

Kohutova, P., Bocquet, F.-X., Henley, E. M. and Owens, M. J. (2016) Improving solar wind persistence forecasts: removing transient space weather events, and using observations away from the Sun-Earth line. Space Weather, 14 (10). pp. 802-818. ISSN 1542-7390 doi: https://doi.org/10.1002/2016SW001447 Available at https://centaur.reading.ac.uk/68635/

It is advisable to refer to the publisher's version if you intend to cite from the work. See Guidance on citing.

Published version at: http://dx.doi.org/10.1002/2016SW001447

To link to this article DOI: http://dx.doi.org/10.1002/2016SW001447

Publisher: American Geophysical Union

All outputs in CentAUR are protected by Intellectual Property Rights law, including copyright law. Copyright and IPR is retained by the creators or other copyright holders. Terms and conditions for use of this material are defined in the End User Agreement. 


\section{CentAUR}

Central Archive at the University of Reading

Reading's research outputs online 


\section{RESEARCH ARTICLE \\ 10.1002/2016SW001447 \\ Key Points: \\ - We improve solar wind persistence \\ models by removing transients, found \\ Improving solar wind persistence forecasts: Removing transient space weather events, and using observations away from the Sun-Earth line} via traits like $\mathrm{He} / \mathrm{H}$ ratios

- Forecasts also improve by using off-Sun-Earth-line data to relax assumptions on coronal stability

- An operational solar wind

persistence forecasting tool

has been implemented at the

Met Office

Correspondence to:

F.-X. Bocquet,

francois.bocquet@metoffice.gov.uk

\section{Citation:}

Kohutova, P., F.-X. Bocquet,

E. M. Henley, and M. J. Owens

(2016), Improving solar wind

persistence forecasts: Removing tran-

sient space weather events, and using observations away from the Sun-Earth line, Space Weather, 14, 802-818, doi:10.1002/2016SW001447.

Received 7 JUN 2016

Accepted 17 SEP 2016

Accepted article online 23 SEP 2016

Published online 22 OCT 2016

@2016. American Geophysical Union and Crown copyright.

This article is published with the

permission of the Controller of HMSO and the Queen's Printer for Scotland.

\author{
Petra Kohutova ${ }^{1,2}$, François-Xavier Bocquet ${ }^{2}$, Edmund M. Henley ${ }^{2}$, and Matthew J. Owens ${ }^{3}$ \\ ${ }^{1}$ Centre for Fusion, Space and Astrophysics, Department of Physics, University of Warwick, Coventry, UK, ${ }^{2}$ Met Office, Fitzroy \\ Road, Exeter, UK, ${ }^{3}$ Space Environment Physics Group, Department of Meteorology, University of Reading, Earley Gate, UK
}

\begin{abstract}
This study demonstrates two significant ways of improving persistence forecasts of the solar wind, which exploit the relatively unchanging nature of the ambient solar wind to provide 27 day forecasts, when using data from the Lagrangian L1 point. Such forecasts are useful as a prediction tool for the ambient wind, and for benchmarking of solar wind models. We show that solar wind persistence forecasts can be improved by removing transient solar wind features such as coronal mass ejections (CMEs). Using CME indicators to automatically identify CME-contaminated periods in ACE data from 1998 to 2011, and replacing these with solar wind from a previous synodic rotation, persistence forecasts improve (relative to a baseline): skill scores for $B_{z}$ a crucial parameter for determining solar wind geoeffectiveness, improve by 7.7 percentage points when using a proton temperature-based indicator with good operational potential. We also show that persistence forecasts can be improved by using measurements away from L1, to reduce the requirement on coronal stability for an entire synodic period, at the cost of reduced lead time. Using STEREO-B data from 2007 to 2013 to create such a reduced lead time persistence forecast, we show that $B_{z}$ skill scores improve by 17.1 percentage points relative to ACE. Finally, we report on implications for persistence forecasts from any future missions to the L5 Lagrangian point and on the successful operational implementation (in spring 2015) of the normal (ACE-based) and reduced lead time (STEREO-based) persistence forecasts in the Met Office's Space Weather Operations Centre, as well as plans for future improvements.
\end{abstract}

\section{Introduction}

The risks posed by geomagnetic activity on human technology have been known for a long time, with the first impacts recorded as early as the 1850s [e.g., Carrington, 1859; Stewart, 1861]. The wide-ranging impacts on modern technology are well documented in scientific literature [e.g., Bolduc, 2002; Dyer, 2002; Sreeja et al., 2014], and detailed assessments have recently been made of the risks for different technology sectors [e.g., Cannon, 2013] and the science and infrastructure required to better understand and mitigate these risks [e.g., Schrijver et al., 2015]. Raising awareness and understanding of the issue in this manner, together with studies on the socioeconomic impacts of space weather [e.g., Hapgood and Thomson, 2010; Schrijver et al., 2014; Gibbs and Bisi, 2015], can hence inform policy responses [e.g., Jonas and McCarron, 2016], to ensure these are appropriate and proportionate.

Accurate forecasting of space weather is a key part of helping mitigate the risk. Various centres around the world help provide this, among them the Met Office's Space Weather Operations Centre (MOSWOC), which has been providing a manned 24/7 operational service since April 2014. This paper presents work developed to help MOSWOC forecasters predict geomagnetic activity arising from the ambient solar wind, and help researchers benchmark other models.

Geomagnetic storms are driven by energy input from the solar wind and can be associated with both the ambient or transient solar wind. Some ambient solar wind features can be geoeffective-for example, corotating interaction regions (CIRs) can trigger geomagnetic substorms and storms. CIRs are regions where the fast solar wind collides with the preceding slow solar wind stream, resulting in compressions which disturb Earth's magnetosphere (possibly indirectly) and are estimated to have been responsible for $\sim 26 \%$ of large 
geomagnetic storms and $\sim 3 \%$ of major storms between 1972 and 2004 [Richardson, 2013]. These CIRs are linked to the position of the coronal holes, where the fast solar wind originates and which evolve slowly over the course of several Carrington rotations, implying some degree of predictability. The other cause of geomagnetic activity is transients in the solar wind in the form of interplanetary coronal mass ejections (ICMEs). The interaction of Earth's magnetosphere with the often-shocked solar wind preceding the flux rope, and with the magnetic field and plasma embedded in the flux rope itself, can all lead to severe geomagnetic storms [e.g., Gosling et al., 1991; Webb et al., 2000].

Owens et al. [2013] used the periodicity of the ambient solar wind to provide a "persistence" forecast with a 27.25 day lead time, using in situ observations of the solar wind made by the Advanced Composition Explorer (ACE) spacecraft at the Lagrangian L1 point. As ACE remains on the Sun-Earth line, it encounters solar wind from corresponding coronal regions on synodic ( 27.25 day) time scales, dictated by the need for a given region to complete a whole solar rotation before its radially traveling solar wind will once again encounter ACE. The ACE-based persistence forecast assumes that the solar wind conditions for the upcoming synodic period will be identical to the ones observed at the current time, as underpinned by an analysis of the autocorrelation functions for a range of solar wind parameters which show clear peaks at multiples of 27.25 days. This persistence model was found to provide improved skill over a climatological model value, making it a valuable tool both as a benchmark against which to assess more complex solar wind models and as a prediction tool in its own right.

The performance of persistence based models is not constant, however-rather, it follows the solar cycle, with improved skill during quiet Sun periods, but degraded skill during the active period in the solar cycle [Owens et al., 2013]. The reason for this is twofold: during active solar cycle periods the coronal features where the solar wind originate are more dynamic, and coronal mass ejections (CMEs) occur more frequently [e.g., McComas et al., 2003; Yashiro et al., 2004]. The observed time series used to produce the forecast contains a record of both the ambient and transient solar wind and in essence assumes that this time series is unaltered one synodic period later. Consequently, more dynamic coronal features reduce the predictability of the ambient solar wind, as do more frequent CME-associated transients - the resulting one-off ICME occurrences at $\mathrm{L} 1$ do not persist through to the next rotation but are unrealistically treated as doing so in a persistence model, unless some filtering is applied.

Section 2 of this paper outlines techniques for the detection of ICME signals in the in situ data used by persistence models. These techniques are then used to identify and remove transients from the forecast and ranked according to their impact on the skill score of the persistence model. Section 3 investigates the use of STEREO data to generate reduced lead time persistence forecast, therefore relaxing the requirement on the stability of the ambient solar wind features. This has been referenced, and section 4 describes the operational persistence forecasts implemented at MOSWOC. Section 5 focuses on the implications of this work for a potential future mission to the Lagrangian L5 point. Finally, some conclusions and future planned work are presented.

\section{Persistence Model With Reduced-Transient Effects}

\subsection{Signatures of Transient Space Weather Events}

Extreme space weather events responsible for geomagnetic storms are characterized by significant increases in the solar wind velocity and magnitude of the magnetic field. Any forecasting model based on the 27 day autocorrelation of the solar wind should therefore account for the presence of these large-amplitude events. Two prominent sources of such conditions are recurrent corotating interaction regions and transient coronal mass ejections. Events that are transient in nature contaminate periodic persistence baselines but can be accounted for if their signatures in the solar wind parameter time series are correctly identified. After specifying contaminated regions of the time series, they can then be removed from the baseline and replaced with a section from the reduced-transient time series on the previous synodic rotation period, which correctly captures the underlying recurrent behavior caused by persisting features in the lower solar atmosphere. In some rare cases, the original (unaltered) time series from the previous rotation period may also have contained transients. But as the transient removal is done on time step-by-time step basis, with reference to the reduced-transient time series, the replacement data will have come from by an uncontaminated section from two synodic rotation periods ago. In even rarer cases, where the original data two synodic rotation periods ago was also contaminated, the replacement data in the reduced-transient time series will come from three synodic periods before - and so on until an uncontaminated section was present. 
The differences in the physical processes responsible for the formation of CMEs and CIRs result in different properties of the plasma associated with each of the two phenomena. Such features, being both compositional and kinematic, can therefore be used to distinguish between signatures of transient and recurrent events in in situ measurements of solar wind parameters. The ICMEs are characterized by several kinematic and compositional signatures as reported by many previous statistical studies [e.g., Gosling, 1990; Neugebauer and Goldstein, 1997; Zurbuchen and Richardson, 2006]. To detect these signatures, we employ a set of criteria based on proton temperature, Fe and $\mathrm{O}$ charge state distributions and He abundance. For this we use measurements taken by operational spacecraft at $1 \mathrm{AU}$, putting emphasis on the present availability of the required data due to potential operational applications.

ICME plasma was found to exhibit proton temperatures $\left(T_{p}\right)$ that are abnormally low compared to the expected temperature $\left(T_{\mathrm{ex}}\right)$ of the ambient solar wind plasma [Gosling et al., 1973]. $T_{\mathrm{ex}}$ follows from the well-established correlation between $T_{p}$ and the solar wind speed $\left(V_{s w}\right)$. In this work we use the empirical $T_{p}-V_{\text {sw }}$ relation established by Lopez [1987]:

$$
\begin{aligned}
& T_{\text {ex }}=\left(0.031 V_{s w}-5.1\right)^{2} \quad V_{s w}<500 \mathrm{~km} / \mathrm{s} \\
& T_{\text {ex }}=0.51 V_{s w}-142 \quad V_{s w}>500 \mathrm{~km} / \mathrm{s}
\end{aligned}
$$

Due to the closed-loop shape of magnetic field lines associated with the ICME structure, the efficiency of the thermal conduction is decreased resulting in cooling of the expanding ICME plasma. The characteristic proton temperatures of the ejecta material are therefore lower than the values typical for the ambient solar wind. We adopt the threshold $T_{p} / T_{\mathrm{ex}} \leq 0.5$ as an indicator of the presence of ICME material in the solar wind [Richardson and Cane, 1995].

The characteristic properties of the origin regions of the ICME plasma are reflected in compositional anomalies in the ICME material. As the coronal density decreases with increasing distance from the Sun, the ionization and recombination time scales for a given element become larger than the solar wind expansion time, leading to freezing-in of the ion charge states. Beyond the freeze-in radius, the ion charge states remain independent of the radial distance from the Sun and are therefore representative of the conditions during solar wind acceleration and ICME formation. Due to the increased abundance of $\mathrm{Fe}^{16+}$ in the material emanating from the hot source regions in the solar atmosphere, the mean iron charge state of the ICME plasma is often elevated to $\left\langle Q_{\mathrm{Fe}}\right\rangle>12$, as compared to the normal value of $\left\langle Q_{\mathrm{Fe}}\right\rangle=10$ for the ambient solar wind [e.g., Lepri et al., 2001; Reinard et al., 2001; Lepri and Zurbuchen, 2004]. In comprehensive surveys of ICME occurrence, about 70\% of ICMEs were found to display elevated $\left\langle Q_{\mathrm{Fe}}\right\rangle$ [Richardson and Cane, 2004], with such enhancement being more likely in faster and flare-related ICMEs [Cane and Richardson, 2003]. Over 95\% of all instances of elevated Fe charge state were found to be associated with ICMEs [Lepri et al., 2001], making it an indicator with a very small proportion of false positive detections.

ICME plasma is also characterized by an elevated $\mathrm{O}^{7+} / \mathrm{O}^{6+}$ abundance ratio [Richardson and Cane, 2004]. Under ambient solar wind conditions, the $\mathrm{O}^{7+} / \mathrm{O}^{6+}$ ratio is anticorrelated with the solar wind speed. This is due to lower freezing-in temperatures in fast solar wind emanating from low-temperature coronal holes [Geiss et al., 1995]. Using recalibrated ACE level 2 data, Kilchenmann [2007] found that this correlation can be modeled by the empirical relation:

$$
O^{7+} / O^{6+}=1.210 \exp \left(-V_{s w} / 200\right)
$$

In the subsequent analysis, we use the $\mathrm{O}^{7+} / \mathrm{O}^{6+}$ ratio given by the above expression as a threshold value for indicating the presence of ejecta material.

Another strong indicator of ICME material is an enhanced $\mathrm{He} / \mathrm{H}$ ratio. The value of $\mathrm{He} / \mathrm{H}$ in ICME material is often greater than 0.08 , as compared to the average solar wind helium abundance of 0.04 [Hirshberg et al., 1972; Neugebauer and Goldstein, 1997]. This signature is present in roughly $50 \%$ of all ICME cases but rarely occurs outside of ICMEs [Yermolaev and Stupin, 1997]. We therefore define He/H $>0.08$ as an ICME indicator.

The above criteria for ICME identification were chosen based on the availability of the required measurements by currently operational spacecraft. We also limited the choice to the indicators that have very small or no probability of occurring outside ICMEs. Additional ICME signatures not used here include, but are not limited to, Mg/O and Ne/O ratios [Richardson and Cane, 2004], smooth rotation of the magnetic field vector 


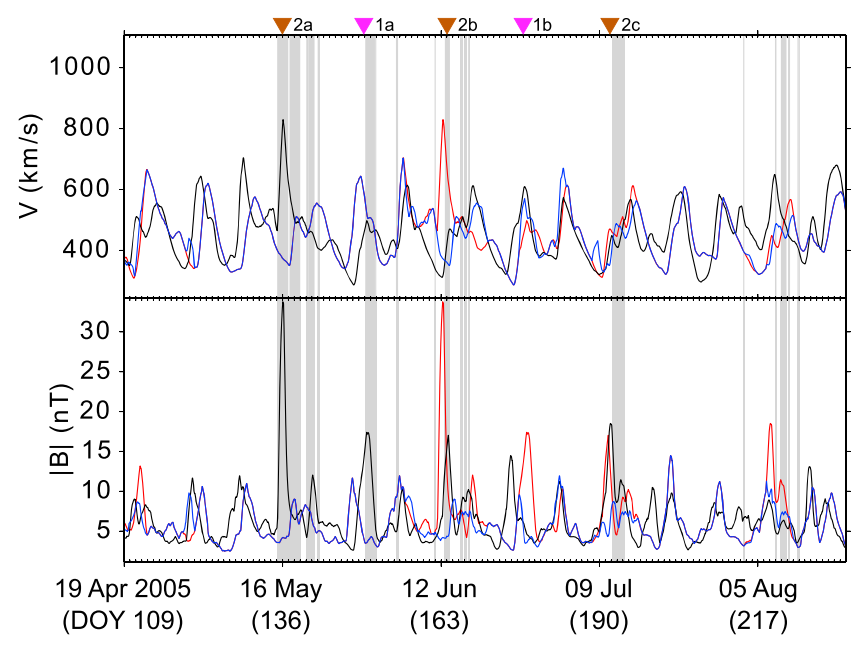

Figure 1. (top) $V_{\mathrm{sW}}$ and $|B|$ (bottom) time series for the ACE observations (black line), standard persistence model (red line), and persistence model with reduced-transient effects (blue line; note this is overplotted on the standard persistence forecast, hiding the latter during times when both forecasts coincide). Grey vertical lines indicate periods of increased mean Fe charge state used by the reduced-transient persistence forecast to try to identify and remove ICME transients. The ICME on 29 May (inverted triangle 1a) causes a false alarm in the standard persistence forecast on 24 June but is correctly identified and removed by the reduced-transient persistence forecast. The reduced-transient forecast also correctly identifies and removes three other ICMEs (arrows 2a, 2b, and 2c), despite their coincidental $\sim 27$ day spacing making it appear as if the reduced-transient forecast has missed event $2 c$ (see text for discussion). Note dates (including day of year) are labeled at 27 day intervals relative to event $2 a$.

caused by the flux rope structure of a subset of ICMEs [e.g., Burlaga et al., 1981], bidirectional particle fluxes [Gosling et al., 1987], and depressed electron temperatures [Montgomery et al., 1974].

It should be noted that the only currently operational source of the solar wind composition measurements in the vicinity of the L1 point, the Solar Wind lon Composition Spectrometer on the ACE spacecraft [Gloeckler et al., 1998], has undergone an age-induced hardware fault in August 2011, leading to the loss of instrument calibration and therefore unavailability of composition data post-2011. The key composition measurements including elemental abundances and charge state information for $\mathrm{Fe}, \mathrm{O}$, and $\mathrm{C}$ have recently become available again; however, they require corrections to address the statistical and calibration issues using the pre-2011 data as a calibration data set (for details see Shearer et al. [2014]).

\subsection{Application of Transient Identification to the 27 Day Persistence Model}

In situ solar wind measurements used in this section were taken by the Advanced Composition Explorer (ACE), operating near the L1 point approximately 0.01 AU sunward of Earth [Stone et al., 1998]. We use magnetic field and plasma measurements from the Magnetic Fields Experiment [Smith et al., 1998] and Solar Wind Electron, Proton, and Alpha Monitor (SWEPAM) [McComas et al., 1998] on board ACE. The required composition data was obtained using measurements by the Solar Wind Ion Composition Spectrometer (SWICS) [Gloeckler et al., 1998]. Hourly averaged level 2 data covering the period from February 1998 to August 2011 was retrieved from the ACE Science Center (http://www.srl.caltech.edu/ACE/ASC/). The range of data used for the analysis was selected to cover the whole period during which constant-calibration data from all three instruments were available simultaneously.

We generate a persistence forecast for seven solar wind parameters: the three components and magnitude of the interplanetary magnetic field; plasma bulk speed and density; and proton temperature. To do this, we use a 27 day persistence model [Owens et al., 2013], based on the $\sim 27$ day autocorrelation of the solar wind parameter time series. To identify the parts of the time series contaminated with transient events, we then use the criteria based on $T_{P},\left\langle Q_{\mathrm{Fe}}\right\rangle, \mathrm{O}^{7+} / \mathrm{O}^{6+}$ and $\mathrm{He} / \mathrm{H}$ described in section 2.1. A time interval with length ranging from 12 to $48 \mathrm{~h}$ either side of the point displaying the nominal ICME signature is then removed from the time series and replaced with an equivalent interval from 27.25 days beforehand.

Figure 1 shows an example of a time series contaminated by an ICME which was subsequently removed (using the $\left\langle Q_{\mathrm{Fe}}\right\rangle$ indicator and a $24 \mathrm{~h}$ interval), resulting in an improved forecast. A rapid increase in magnetic field magnitude was observed by ACE (black line in Figure 1) on the 29 May 2005 (indicated by inverted 
triangle 1a). This causes a false alarm in the standard persistence forecast (red line) on 24 June (inverted triangle $1 \mathrm{~b}$ ). Since this event was accompanied by an elevated iron charge state, it was classified as ICME and removed from the reduced-transient persistence forecast (blue line) by the process above, resulting in an overall improvement of the forecast skill. It is also interesting to note a triplet of events occurring on the 16 May, 13 June, and 10 July (arrows 2a, 2b, and 2c). The first event (2a) shows a clear increase in magnetic field magnitude and plasma bulk speed, characteristic of an ICME, and is accompanied by an elevated iron charge state and is hence removed. As in the previous example, it is seen that this filtering results in this event not appearing in the reduced-transient forecast 27 days later, on the 12 June (whereas it does appear in the normal persistence forecast). This is expected to be a desirable behavior; however, it is seen that a second event $(2 \mathrm{~b})$ is observed at roughly the same time and that a third event $(2 \mathrm{c})$ occurs roughly a further 27 days later, on 10 July. Both events are also accompanied by elevated iron charge states - this results in event $2 \mathrm{~b}$ being removed for the reduced-transient forecast and hence not present near event $2 c$ - thus apparently performing worse than the standard persistence forecast, which seems to predict event $2 c$. The near 27 day spacing between these events may initially seem suggestive of CIR effects, and a false positive for the iron-charge-based reduced-transient forecast for event $2 \mathrm{~b}$. However, closer examination suggests this is not the case: not only are all three events ( $2 a, 2 b$, and $2 c)$ accompanied by elevated iron charge states expected for ICMEs, but they are independently listed as being associated with ICMEs in the Richardson and Cane [2010] catalog. Thus, we conclude that the $\sim 27$ day spacing between these events is coincidence and that these events are indeed all transients, correctly being filtered out by the reduced-transient persistence forecast.

The model performance can be assessed by evaluating the forecast skill against a random reference model, or baseline [Spence et al., 2004]. The skill of the model is based on the Mean Square Error (MSE) of the forecast and is defined as

$$
\text { Skill }=100\left(1-\frac{M_{\text {SE }}}{M_{\text {mod }}}\right)
$$

where $\mathrm{MSE}_{\text {mod }}$ is the mean square error between the model and the observation and $\mathrm{MSE}_{\text {ref }}$ is the mean square error between the reference model and the observation. A forecast skill of 100 corresponds to a perfect forecast, whereas negative skill means that the forecast performance of the model is worse than that of a random forecast. In order to create a reference model with sufficient degree of variability but zero autocorrelation on all time scales, a random reference model with the same bulk statistical properties as the observed time series is chosen. Using the method described by Owens et al. [2013], the reference time series with the required properties is obtained by creating a cumulative distribution function (CDF) for each solar wind parameter. Subsequently, a random number generator is used to generate random values between 0 and 1 which are then assigned the corresponding parameter values from the CDF. The overall skill score is evaluated for each parameter by considering mean values of MSE for both model and baseline. The skill scores of the persistence model before and after the transient removal are shown in Table 1.

As can be seen, not all indicators lead to increases across all individual parameters - for example, when using the $T_{p}$ indicator alone, the skill of the reduced-transient forecast for $V_{s w}$ is lower than the original persistence forecast. This agrees with the results of Wardle [2013], who used OMNI data from 1996 to 2011 to generate reduced-transient persistence forecasts for solar wind speed and also found a skill decrease when using proton temperature data to remove transients. Wardle [2013] further found that using $T_{\mathrm{ex}} / T_{p}$ threshold value lower than that used here (the $T_{\mathrm{ex}} / T_{p}=0.5$ threshold proposed by Richardson and Cane [1995]) results in an improved performance of the new forecast. This has not been investigated further here, as this indicator does not lead to the highest increase in skill. Nevertheless, this indicator will merit further attention, as $T_{p}$-based filtering is operationally feasible (see later discussions in sections 4 and 5.3) and does lead to increases in $B_{z}$ and average skill (of 7.7 and 3.0 percentage points, respectively, for the best performing $48 \mathrm{~h}$ time interval.)

The average increase in skill with respect to the standard persistence model is highest when using all indicators combined and removing a $12 \mathrm{~h}$ time interval either side of the event as described above. In this case, the skill increase averaged over all solar wind parameters considered in this section is 8.0 percentage points. However, there is a considerable difference in the skill increase for individual parameters, with the smallest increase of 0.6 percentage points in $V_{\mathrm{sw}}$ and the maximum skill increase of 16.7 percentage points for the $B_{z}$ component of the magnetic field. A comparable 7.9 percentage point increase in average performance can also be obtained from a single indicator, $\left\langle Q_{\mathrm{Fe}}\right\rangle$, when removing 24 hour intervals: this yields similar changes in individual parameters, $V_{s w}$ also having the smallest skill increase (1.7 percentage points), and $B_{z}$ also having 
Table 1. Forecast Skill Scores From ACE Data (1998-2011) for the Persistence Model With Reduced-Transient Effects Using Different ICME Indicators, As Well As Durations (in Hours) for the Contaminated Time Interval $\Delta t_{c}$, Replaced by Solar Wind From a Previous Rotation ${ }^{\mathrm{a}}$

\begin{tabular}{|c|c|c|c|c|c|c|c|c|c|c|c|c|c|c|c|c|c|}
\hline Indicator & $\Delta t_{c}$ & $B_{x}$ & $\Delta_{S}$ & $B_{y}$ & $\Delta_{S}$ & $B_{z}$ & $\Delta_{S}$ & $B$ & $\Delta_{S}$ & $N_{p}$ & $\Delta_{S}$ & $V_{\mathrm{sW}}$ & $\Delta_{S}$ & $T_{p}$ & $\Delta_{S}$ & $\bar{S}$ & $\Delta_{S}$ \\
\hline None (control) & - & 36.2 & - & 27.9 & - & 3.5 & - & 26.0 & - & 32.3 & - & 52.0 & - & 34.3 & - & 30.3 & - \\
\hline $\mathrm{Fe}$ & 48 & 38.3 & 2.1 & 33.7 & 5.8 & 20.1 & 16.6 & 38.1 & 12.1 & 38.4 & 6.1 & 52.1 & 0.1 & 41.7 & 7.4 & 37.5 & 7.2 \\
\hline $\mathrm{He}$ & 48 & 36.8 & 0.6 & 32.7 & 4.8 & 17.1 & 13.6 & 35.8 & 9.8 & 36.9 & 4.6 & 51.6 & -0.4 & 37.6 & 3.3 & 35.5 & 5.2 \\
\hline 0 & 48 & 38.9 & 2.7 & 32.9 & 5.0 & 16.1 & 12.6 & 36.7 & 10.7 & 37.2 & 4.9 & 53.4 & 1.4 & 39.0 & 4.7 & 36.3 & 6.0 \\
\hline $\mathrm{Tp}$ & 48 & 36.9 & 0.7 & 31.5 & 3.6 & 11.2 & 7.7 & 31.5 & 5.5 & 36.7 & 4.4 & 50.5 & -1.5 & 34.9 & 0.6 & 33.3 & 3.0 \\
\hline $\mathrm{Fe}+\mathrm{He}+\mathrm{O}+\mathrm{Tp}$ & 48 & 35.5 & -0.7 & 32.8 & 4.9 & 21.9 & 18.4 & 37.6 & 11.6 & 38.1 & 5.8 & 49.4 & -2.6 & 40.1 & 5.8 & 36.5 & 6.2 \\
\hline $\mathrm{Fe}$ & 24 & 40.2 & 4.0 & 35.0 & 7.1 & 19.3 & 15.8 & 39.1 & 13.1 & 38.5 & 6.2 & 53.7 & 1.7 & 41.8 & 7.5 & 38.2 & 7.9 \\
\hline $\mathrm{He}$ & 24 & 38.1 & 1.9 & 33.2 & 5.3 & 15.9 & 12.4 & 34.6 & 8.6 & 36.4 & 4.1 & 52.0 & 0.0 & 36.5 & 2.2 & 35.2 & 4.9 \\
\hline $\mathrm{O}$ & 24 & 38.9 & 2.7 & 33.0 & 5.1 & 14.8 & 11.3 & 35.7 & 9.7 & 36.9 & 4.6 & 53.6 & 1.6 & 36.5 & 2.2 & 35.6 & 5.3 \\
\hline $\mathrm{Tp}$ & 24 & 37.5 & 1.3 & 31.0 & 3.1 & 9.6 & 6.1 & 30.6 & 4.6 & 35.1 & 2.8 & 51.3 & -0.7 & 34.9 & 0.6 & 32.9 & 2.5 \\
\hline $\mathrm{Fe}+\mathrm{He}+\mathrm{O}+\mathrm{Tp}$ & 24 & 38.5 & 2.3 & 35.2 & 7.3 & 21.3 & 17.8 & 39.1 & 13.1 & 38.7 & 6.4 & 51.8 & -0.2 & 41.3 & 7.0 & 38.0 & 7.7 \\
\hline $\mathrm{Fe}$ & 12 & 41.0 & 4.8 & 35.0 & 7.1 & 18.2 & 14.7 & 38.6 & 12.6 & 38.8 & 6.5 & 54.0 & 2.0 & 40.4 & 6.1 & 38.0 & 7.7 \\
\hline $\mathrm{He}$ & 12 & 37.7 & 1.5 & 32.0 & 4.1 & 12.5 & 9.0 & 32.3 & 6.3 & 35.6 & 3.3 & 51.7 & -0.3 & 34.8 & 0.5 & 33.8 & 3.5 \\
\hline 0 & 12 & 38.8 & 2.6 & 32.4 & 4.5 & 13.9 & 10.4 & 34.7 & 8.7 & 35.7 & 3.4 & 53.8 & 1.8 & 35.9 & 1.6 & 35.0 & 4.7 \\
\hline $\mathrm{Tp}$ & 12 & 36.8 & 0.6 & 30.2 & 2.3 & 7.3 & 3.8 & 28.6 & 2.6 & 34.4 & 2.1 & 51.4 & -0.6 & 35.0 & 0.7 & 32.0 & 1.6 \\
\hline $\mathrm{Fe}+\mathrm{He}+\mathrm{O}+\mathrm{Tp}$ & 12 & 40.0 & 3.8 & 35.8 & 7.9 & 20.2 & 16.7 & 39.5 & 13.5 & 40.2 & 7.9 & 52.6 & 0.6 & 40.0 & 5.7 & 38.3 & 8.0 \\
\hline
\end{tabular}

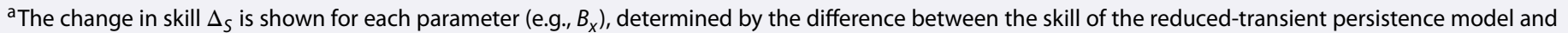
the standard persistence model (where no transient filtering is performed). For each $\Delta t_{c}$, the mean change in skill over all parameters $\bar{S}$ is shown, together with the change in skill $\Delta_{S}$ relative to the standard persistence model mean. Improvements in skill (relative to the standard persistence model) are shown in bold.

the maximum skill increase (15.8 percentage points). Given the similarity in average and $B_{z}$ skill increase between the combination of all indicators and the (marginally worse) individual $\left\langle Q_{\mathrm{Fe}}\right\rangle$ indicator, for simplicity the rest of this paper concentrates on $\left\langle Q_{\mathrm{Fe}}\right\rangle$ results.

The small change in the forecast skill in the solar wind speed prediction is in fact expected; due to the action of the drag force exerted by the ambient solar wind during ICME propagation, the ICME speeds evolve toward the ambient solar wind speed. At $1 \mathrm{AU}$, only a small percentage of ICMEs have speeds significantly greater [e.g., Yashiro et al., 2004; Manoharan, 2006; Vršnak et al., 2008]. Since the speeds of the majority of ICMEs are not largely different from the ambient solar wind speed, removing such events will have only small effect on the predicted speed time series, given the length of the data set used. This is further supported by the fact that even prior to transient removal, the forecast skill for the solar wind speed is $52 \%$, i.e., significantly higher than for other parameters. Work to determine the causes of this has not been performed; however, this is unlikely to be due to the plasma data cadence being lower than that of magnetic field data, given hourly averaged magnetic and plasma data have been used here; rather, this is likely to be due to the lower variability (on short time scales) of solar wind speed compared to magnetic field measures [e.g., Wicks et al., 2010].

On the other hand, ICME removal has a larger impact on the magnetic field: ICMEs are often characterized by long-term enhancements of the north-south interplanetary magnetic field component $B_{z}$, geoeffective impacts coming largely from large negative (southward) $B_{z}$ values over extended periods of time. After removal of ICMEs, these enhancements in the $B_{z}$ time series are no longer present and therefore will not generate false alarms in the future. The advantage of this method is that it filters out the transient ICME $B_{z}$ disturbances but leaves the recurring $B_{z}$ disturbances associated with CIRs [Burlaga and Lepping, 1977] unchanged, so these CIR-associated are forecast for the next solar rotation.

Figure 2 shows the overall improved prediction in the baseline value of the $B_{z}$ magnetic field component coming from the reduced-transient persistence forecast (using the $\left\langle Q_{\mathrm{Fe}}\right\rangle$ indicator and $24 \mathrm{~h}$ intervals). While the possibility of the misidentification of recurring events as ICMEs cannot be completely eliminated, there are many examples of successful ICME removal and CIR retention. Illustrative examples are shown for 2004: brown inverted triangles indicate ICMEs and their subsequent appearance in the standard persistence forecast, where they have correctly been rejected by the reduced-transient forecast, while pink inverted 


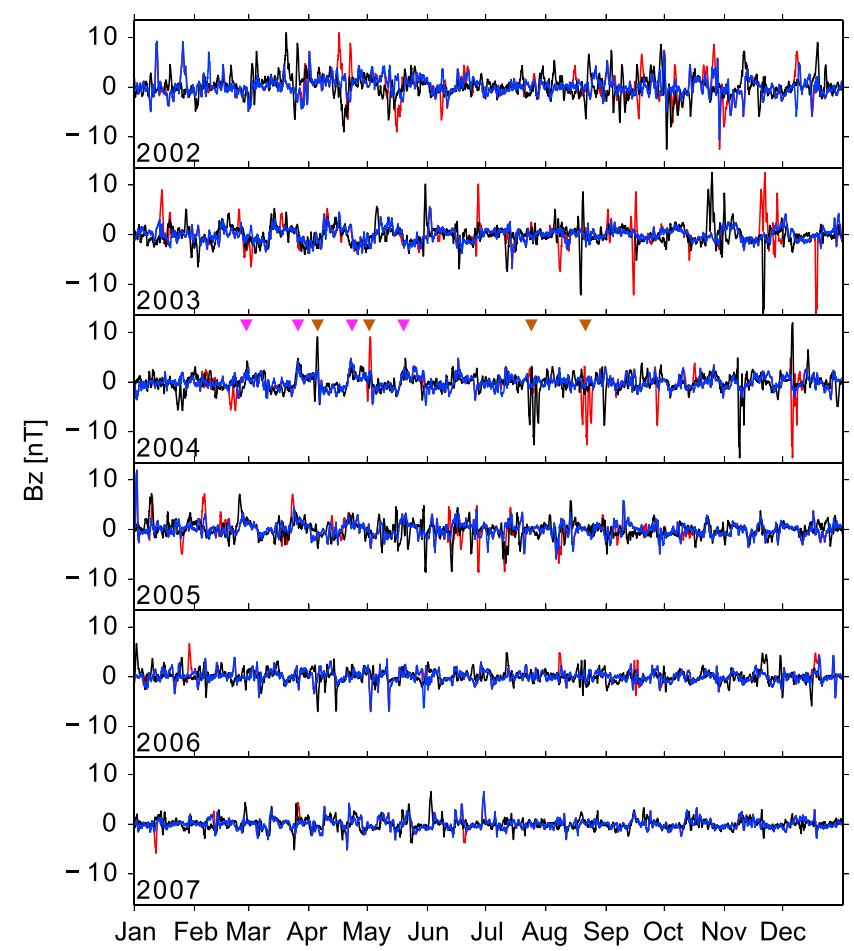

Figure 2. Increase in the forecast skill for the $B_{z}$ component of the magnetic field. ACE observations are shown in black, and the normal persistence model is shown in red. The persistence model with reduced-transient effects (blue, using Fe (24 h) indicator) shows better agreement with observations, as it does not incur a double penalty due to transient features (brown arrows show examples in 2004 where this has occurred for the standard persistence forecast) but is still able to predict repeating structures with $\sim 27$ day periodicity (highly likely to be associated with CIRs; examples in 2004 shown with pink inverted triangles).

triangles show repeating structures with $\sim 27$ day periodicity (highly likely to be associated with CIRs) which have correctly been retained by the reduced-transient forecast.

The overall impact of this iron charge-based transient removal technique is an increase in the $B_{z}$ forecast skill from $3.5 \%$ to $19.3 \%$ (see Fe $(24 \mathrm{~h}$ ) entry in Table 1 ). This is likely to be due largely to the physical factors identified above (removing ICME false alarms, correctly retaining CIR effects) but may also be somewhat mathematical - the resulting flattening of $B_{z}$ time series may also help improve skill, much as a flat forecast can have better skill than a mistimed forecast [Owens et al., 2013]. Further work is needed to determine the precise contribution of each effect.

Nevertheless, these results are promising, since the southward IMF component $B_{z}$ is a key parameter in determining space weather geoeffectiveness - the improved prediction of the baseline value of $B_{z}$ seen in Figure 2 represents the main advantage of the persistence model with reduced space weather effects.

\section{Reduced Lead Time Persistence Model}

In addition to using in situ measurements for solar wind forecasting at the same location in the next synodic rotation period, they can also be used to predict solar wind conditions at a different location in the Parker spiral, with the forecast lead time depending on the heliographic longitudinal separation between the two reference points. We extend the 27 day solar wind persistence model for space weather forecasting at the L1 point established by Owens et al. [2013] to other locations in the Parker spiral by using STEREO spacecraft observations. The STEREO mission consists of two identical spacecraft on very similar $\sim 1 \mathrm{AU}$ orbits as Earth; STEREO-A launched azimuthally ahead of the Earth and STEREO-B launched trailing behind the Earth [Kaiser, 2005]. Orbital differences with Earth mean the longitudinal separation between each spacecraft and the Earth increases at a rate of $\sim 22.5^{\circ}$ per year (as both spacecraft separate by $\sim 45^{\circ}$ per year [Kaiser, 2005]). For the time interval considered here, STEREO-B was located azimuthally behind Earth; we therefore use the in situ measurements taken by STEREO-B to develop a reduced lead time persistence model for solar wind 


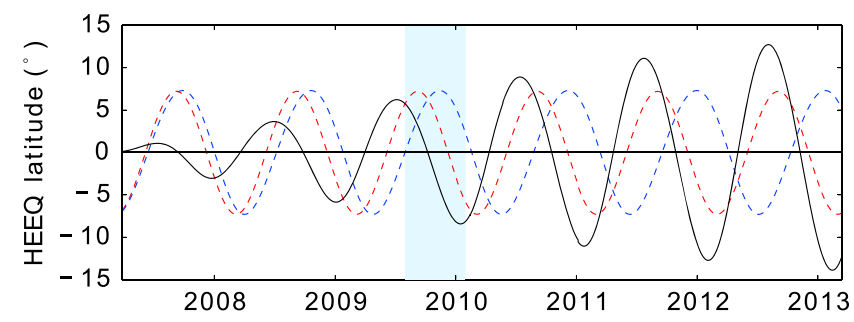

Figure 3. ACE (red) and STEREO-B (blue) heliographic latitude as a function of time. The black line shows the heliolatitude difference between the two spacecraft. The blue shaded section corresponds to the period when STEREO-B was in the vicinity of L5.

conditions at L1 as this effectively encounters the solar wind conditions which will affect Earth shortly after, assuming corotation of the solar wind.

The orbits of ACE and both STEREO spacecraft lie in the ecliptic plane. Due to the tilt of the solar rotation axis with respect to the ecliptic, the heliographic latitude of STEREO-B is not the same as that of ACE and their latitudinal separation varies with time, with a period of approximately a year (Figure 3). This latitudinal separation means ACE and STEREO may sample qualitatively different solar wind streams, even during periods of perfect solar wind corotation. In fact, latitudinal separation is expected to have the greatest effect on such reduced lead time persistence forecasts when the coronal magnetic field is most structured by heliolatitude, which is primarily around solar minimum when the assumption of corotation is strongest. This effect is expected to correlate with the absolute latitudinal separation of the spacecraft, hence with a period of approximately 6 months. This heliolatitude effect limits the extent to which a spacecraft (e.g., STEREO-B here) off the Sun-Earth line can be expected to improve on ACE. The longitudinal separation, on the other hand, acts as an advantage, since using such a spacecraft effectively reduces the requirement on the stability of solar wind conditions, by reducing the lead time of the persistence forecast from the 27 days required when using ACE. As is shown below, this "sacrifice" on lead time results in better overall forecast performance, especially during periods of high solar variability. While 27 day lead times may be desirable, these are not a key requirement by most operators. These will benefit more from increased skill in the 5 day forecast.

In order to generate the reduced lead time persistence forecast, we use the magnetic field measurements taken by the magnetometer from the In-situ Measurements of Particles And CME Transients instrument suite [Acuña et al., 2007] and the measurements of proton temperature, density, and plasma bulk speed by the Plasma and Supra-Thermal Ion Composition Investigation instrument [Galvin et al., 2008] on board STEREO-B. The hourly averaged level 2 data covering a period from the beginning of STEREO operation in March 2007 until March 2013 were retrieved from the UCLA Space Science Center (http://aten.igpp.ucla.edu/ssc/stereo/). The STEREO-B measurements of the magnetic field vector were transformed from spacecraft-centered RTN coordinates to GSM coordinate system and subsequently rotated by the azimuthal angle $\Theta$ between the spacecraft and the Earth, with the Sun at the vertex. We then offset the observations by a fraction of the synodic rotation period corresponding to the current position of STEREO-B. The time offset $\Delta t$ applied to the observation time series is given by

$$
\Delta t=\frac{T_{\text {rot }}}{360^{\circ}} \lambda(t)
$$

where $T_{\text {rot }}$ is the synodic rotation period and $\lambda(t)$ is the heliographic longitude of the spacecraft relative to Earth.

Table 2 summarizes the values of linear cross-correlation coefficients and skill scores evaluated for multiple solar wind parameters for both ACE- and STEREO-based persistence forecasts. Note that STEREO evaluation is done relative to ACE baselines (e.g., for the CDF described above), as the objective is to forecast conditions affecting Earth. The STEREO forecast performs better for all parameters except for proton density and temperature, where the analysis suggests better correlation of the STEREO-B based persistence model with the observation time series but worse forecast skill than the standard persistence model. This is caused by the fact that the correlation coefficient is independent of constant offsets or scaling. It therefore provides a measure of how well the evolution of the two time series agrees, whereas the MSE-based assessment tools 
Table 2. Skill Scores and Linear Correlation Coefficients for ACE and STEREO-B-Based Persistence Models for Various Solar Wind Parameters Over the Period March 2007 to March $2013^{a}$

\begin{tabular}{lcccccc}
\multicolumn{3}{c}{ ACE } & \multicolumn{5}{c}{ STEREO-B } \\
\hline Parameter & Skill & $r_{L}$ & Skill & $\Delta_{S}$ & $r_{L}$ & $\Delta_{r}$ \\
$B_{x}$ & 29.1 & 0.50 & 40.9 & $\mathbf{1 1 . 8}$ & 0.56 & $\mathbf{0 . 0 6}$ \\
$B_{y}$ & 20.7 & 0.42 & 30.4 & $\mathbf{9 . 7}$ & 0.45 & $\mathbf{0 . 0 3}$ \\
$B_{z}$ & 4.3 & 0.14 & 21.4 & $\mathbf{1 7 . 1}$ & 0.18 & $\mathbf{0 . 0 4}$ \\
$|B|$ & 16.6 & 0.24 & 24.8 & $\mathbf{8 . 2}$ & 0.33 & $\mathbf{0 . 0 9}$ \\
$N_{p}$ & 54.2 & 0.41 & 23.2 & -31.0 & 0.41 & 0.00 \\
$V_{p}$ & 56.3 & 0.59 & 65.4 & $\mathbf{9 . 1}$ & 0.70 & $\mathbf{0 . 1 1}$ \\
$T_{p}$ & 44.8 & 0.45 & 29.3 & -15.5 & 0.48 & $\mathbf{0 . 0 3}$ \\
\hline
\end{tabular}

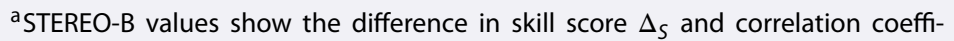
cient $\Delta_{r}$ relative to ACE values. Bold numbers show where these differences indicate the STEREO-B-based persistence forecast improves over the ACE-based persistence forecast.

are only concerned with the agreement in the magnitude of the predicted and observed parameter. The disagreement between the two assessment methods can occur when comparing measurements made by two different instruments, where differences in the calibration are liable to lead to different scaling and offsets. This is especially likely given the complex calibration required to determine the bulk plasma parameters $V_{\text {sw' }}$ $T_{p}$, and $N_{p}$ from electrostatic deflection analyzers [Paschmann and Daly, 1998, chapter 6], especially given those used on ACE are not designed to be identical to those on STEREO [McComas et al., 1998; Galvin et al., 2008]. It is notable that the only decrease in skill for STEREO-B (compared to ACE) occurs in the $N_{p}$ and $T_{p} ; V_{\text {sw }}$ shows an increase in skill (consistent with the magnetic field results). The countervailing skill score behavior between the various plasma moments may be due to intercalibration issues between ACE and STEREO-B, $V_{s w}$ not exhibiting this due to its relative robustness to calibration challenges [Paschmann and Daly, 1998, chapter 6]. Further investigation is needed, however, to determine if intercalibration between ACE and STEREO-B is indeed responsible for this behavior. If this is indeed due to instrument calibration, it will likely be possible to use simple regressions to "bias correct" the STEREO-B observations to match the ACE observations.

Due to the increasing longitudinal separation between the two spacecraft, the performance of the reduced lead time persistence model is expected to decrease due to the increased time over which corotation is assumed to hold. In addition, the variability of the solar wind parameters is subject to the phase of the solar cycle. In order to fully capture the long-term evolution of any performance measures, we evaluate the performance indicators on a rolling basis. Figure 4 shows the variation in the forecast skill and the correlation coefficient, evaluated for each parameter using a 1 year rolling window (right aligned, i.e., at each time step, using the preceding year's data for each parameter to determine the skill and correlation coefficient).

The value of the correlation coefficient for the 27 day ACE persistence forecast varies significantly during the 7 year period, with the performance of the ACE persistence forecast being best during 2008-2009, corresponding to the period of the solar activity minimum. The correlation decreases as the Sun enters the solar maximum, and the degree to which the solar wind can be assumed to corotate is decreased. In addition to the solar cycle variation, the performance of the STEREO-B-based persistence model (relative to ACE conditions relevant to Earth) is influenced by two further factors; the increasing azimuthal angle $\Theta$ and varying heliographic latitudinal separation between the spacecraft and the Earth. As the STEREO-B azimuthal angle $\Theta$ increases from $0^{\circ}$ in March 2007 to $140^{\circ}$ in March 2013, the spacecraft slowly loses advantage over ACE in terms of the requirement on the solar wind stability time scale. Treating the rolling correlation of the ACE persistence model as a measure of the model performance variability due to the changing solar cycle phase, we subtract it from the STEREO-B correlation time series to obtain the overall trend in the STEREO-B forecast performance due to azimuthal angle $\Theta$ and heliographic latitude effects. This results in a weak downward trend, where the decrease in the model performance is most pronounced during the first 2 years of the analyzed data set when the azimuthal angle $\Theta$ varies from $0^{\circ}$ to $50^{\circ}$. The performance of the model post-2009 stays roughly constant. 

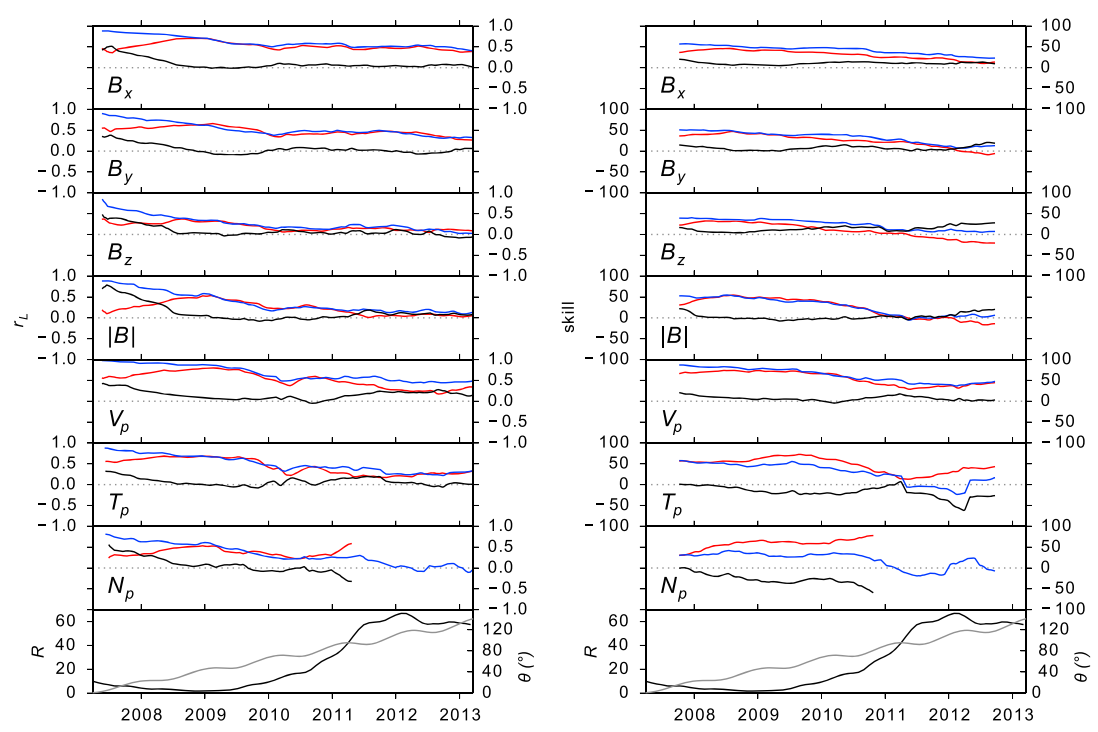

Figure 4. Time evolution of (left column) correlation coefficients and (right column) skill scores for ACE (red) and STEREO-B (blue) persistence forecasts evaluated using a 1 year rolling window. The black line shows the overall trend in the STEREO-B forecast performance after subtracting the solar cycle dependence. The grey line in the eighth row shows the STEREO-B azimuthal angle $\Theta$. The black line shows the monthly sunspot number $R$ as a reference of the solar cycle phase. Sunspot data were obtained from the World Data Center SILSO, Royal Observatory of Belgium, Brussels.

For most parameters, there is no obvious variation in the trend, suggesting that the small variation in heliographic latitude is a relatively unimportant effect compared to the increasing azimuthal angle $\Theta$. However, we have further analyzed the effect of the varying latitudinal separation on the performance of STEREO-B persistence model by taking a Fourier Transform of the detrended STEREO-B correlation time series evaluated using a 30 day rolling window. Figure 5 shows the normalized power spectra of the STEREO-B correlation and of the square of the latitudinal difference time series. The latter quantity varies with a period of approximately 6 months (as discussed in Figure 3). Most solar wind parameters show limited variation on this period,
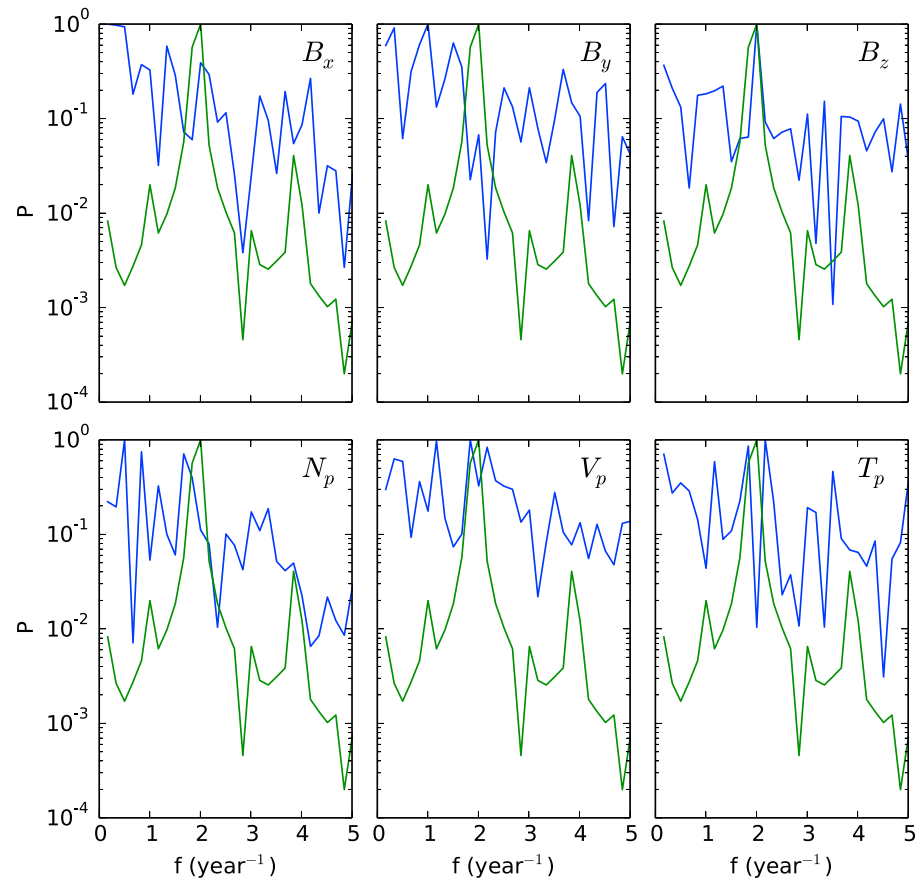

Figure 5. Normalized power spectra of the periodically varying latitudinal separation between ACE and STEREO-B (green), and equivalent spectra (blue) for the (detrended) STEREO-B forecast model correlation time series. 
suggesting latitudinal variation has negligible impact on the associated STEREO-B model performance. The correlation power spectrum of $B_{z}$ component of the magnetic field however does show a prominent peak near this $\sim 6$ month frequency for absolute latitudinal separation variations. This suggests the presence of large heliolatitudinal gradients in $B_{z}$, which may degrade the performance of the STEREO-B model during periods of large latitudinal separations between the ACE and STEREO-B spacecraft.

Re-examining the $B_{z}$ rolling correlation coefficients and skill scores in Figure 4 carefully, some minor semiannual oscillations can be seen in the correlations, notably toward the latter part of the period (around 2011), when Figure 3 shows latitudinal separations are largest. However, these oscillations are small and are not visible in skill, supporting the view above that this is a comparatively minor effect.

\section{Operational Tool}

Since the studies in the previous section were performed, the 27 day and the reduced lead time persistence models have been adapted and implemented operationally in spring 2015. They provide MOSWOC forecasters with a forecast tool that generates real-time plots of solar wind parameters for the upcoming 27 days using beacon data for seven solar wind parameters. The data used to generate these forecasts consists of hourly resolution ACE beacon data provided by NOAA's Space Weather Prediction Centre (SWPC) and 10 min resolution data available from NASA's STEREO Science Center, averaged to hourly resolution. At time of writing (April 2016) STEREO-A data are used as STEREO-A is currently in the ideal Earth-trailing position STEREO-B was in for the studies performed in section 3.

The forecast tool, shown in Figure 6, provides time series plots of the individual components of the magnetic field in GSM coordinates, magnetic field magnitude, plasma bulk speed, proton density, and ion temperature with the lead time of 27.25 days for ACE forecast and currently (April 2016) 13 days for STEREO-A forecast. The mean square errors between the forecast and the observation are adopted as a measure of uncertainty of the forecast for the upcoming period and are represented as a shading surrounding the forecast timeline; forecasts and observations from the previous rotation are also shown to allow forecasters to judge whether there are systematic errors potentially affecting the current period (e.g., if the relevant coronal holes are far from the solar equator; differential solar rotation means the lead times used above are less appropriate). To make the tool simple and versatile for forecasters, options are available to hide STEREO data, show only magnetic field magnitude and solar wind velocity, and customize the forecast period to shorter intervals.

There are two important aspects to consider in this operational implementation. First is the fact that the skill scores reported in sections 2.2 and 3 are unlikely to represent the performance of the operational tool. This is because the beacon data used for operational purposes are of lower quality than the level 2 (processed) data analyzed in the previous sections. Therefore, there will be some differences in the statistical properties of these operational forecasts. In particular, the majority of the ACE beacon values for the proton density and temperature have been deemed as unreliable (possibly due to an issue with SWEPAM calibration) and removed from the level 2 data resulting in the lack of data points. Consequently, it is important to verify that the outcomes of the skill-score-based performance analysis discussed in the previous sections are valid for real-time-based models as well. In order to do this, the beacon data used as forecast tool input are being archived for the purpose of future investigation.

The second point is that the transient removal process described in section 2.2 is not being applied operationally. As mentioned in section 2.1, the SWICS instrument aboard ACE has suffered hardware damage in August 2011 (see SWICS webpage) and the processing of the heavy ion data has been altered after that date and is now being archived as SWICS 2.0. This new data set has not yet been analyzed to assess whether it is also suitable for the transient removal —in the meantime, the 27 day persistence forecast has been implemented without transient removal.Two practical issues with using SWICS data for operational purposes are the fact that the data are not available in real time and the fact that ACE has already exceeded its expected lifetime and might stop providing data anytime. Furthermore, the Deep Space Climate ObserVatoRy (DSCOVR) mission to replace ACE will also soon become the primary source for real-time solar wind monitoring data. Consequently, we currently intend to switch the data source for the 27 day persistence model from ACE to DSCOVR, but as DSCOVR does not contain an instrument to analyze heavy ions, it will not be possible to implement the transient removal technique using the mean iron charge state (the best individual indicator found in section 2.2). Instead, we intend to implement transient removal using the proton temperature indicator, 


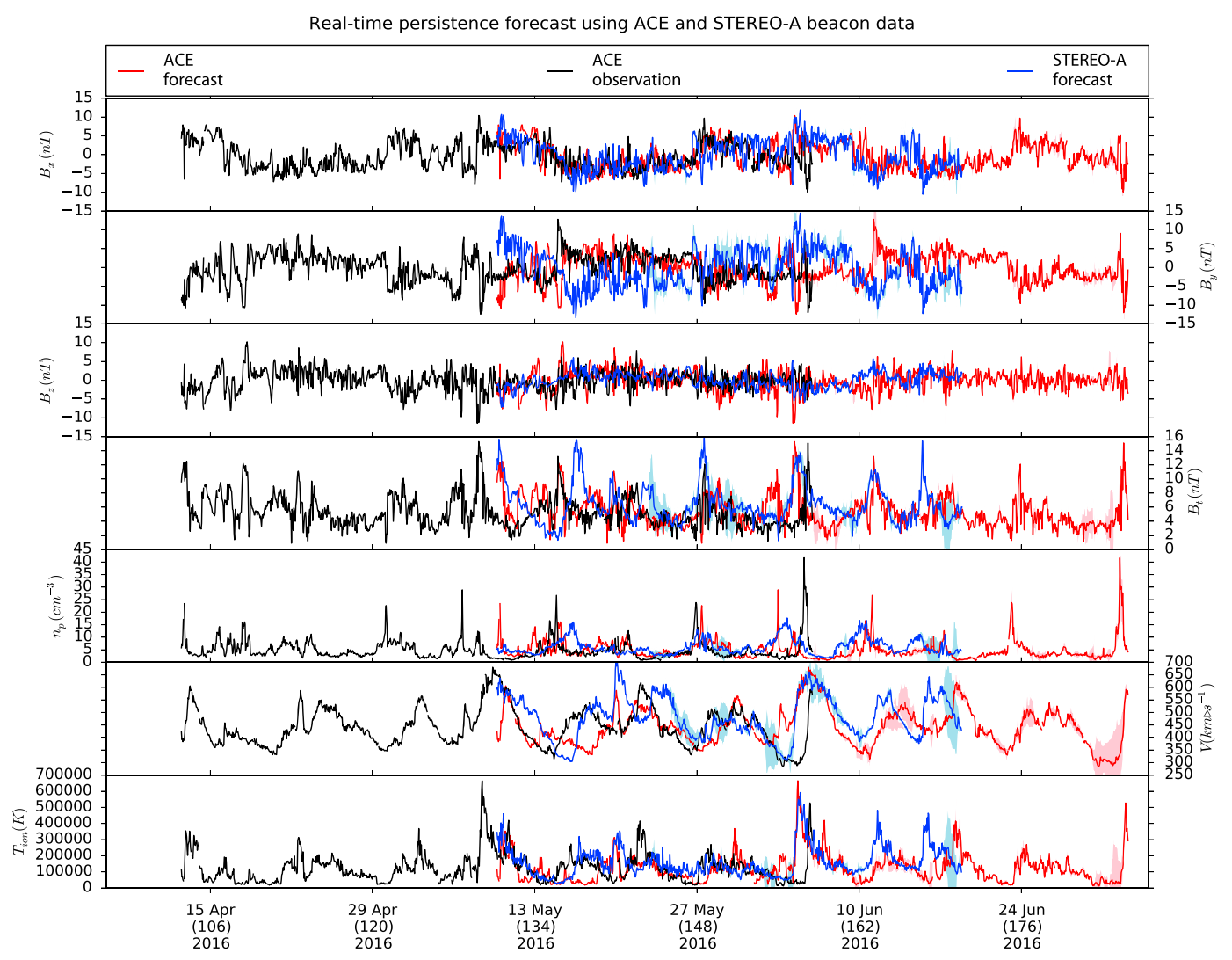

Figure 6. Met Office operational solar wind persistence model output for 2016-04-19T12Z. Time series from top to bottom show $B_{x}, B_{y}, B_{z}$, and $|B| ; n_{p}, V_{s w}$, and $T_{p}$. ACE-based persistence forecast results (red) are shown for the next 27 days, but also (with ACE observations, black) for the last 27 days, so MOSWOC forecasters can judge the forecast's recent performance. STEREO-based persistence forecasts are also shown in blue. Shading around each forecast corresponds to the uncertainty in the forecast, as evaluated by the disagreement between forecast and observations over the last 27 days. Note that the apparent absence of shading is due to the fact that in some cases, the uncertainty is quite small and the shading not readily noticeable. Also note that CME filtering has not been implemented, due to data availability issues.

as this will be available in real time from the DSCOVR Solar Wind Plasma Sensor and Magnetometer (PlasMag) instrument [Samuelson, 2015]. This will be backed up with an investigation of the best $T_{\mathrm{ex}} / T_{p}$ threshold to apply, as discussed in section 2.2. Once this has been done, we intend to implement real-time determination of the rolling skill score, and display this, to help forecasters judge how well the persistence forecast has been performing recently.

\section{Implications for an L5 Mission}

The results in section 3 have shown that for persistence forecasts, there is benefit from observing the solar wind azimuthally away from the Sun-Earth line, as this means less than a full solar rotation is needed before the observed solar wind stream nominally encounters the Earth, thus diminishing the requirement for this stream to remain constant (for such observations to lead to a skillful persistence forecast) by comparison with observations on the Sun-Earth line, which require a full solar rotation (and hence a longer period of constancy).

The sacrifice made for this is reduced lead time. The balance of benefits between increased accuracy and lead time will depend on the forecast application; in principle, different sectors might benefit from persistence forecasts issued by spacecraft theoretically present at a wide range of azimuthal separations from the Sun-Earth line. In practice, however, it is worth noting that the STEREO-B case studied here is notable, as its orbit meant it gives some indication of the likely increase in persistence forecast skill (relative to observations on the Sun-Earth line) which may be expected from an azimuthally offset spacecraft with a good case for being commissioned - an operational space weather monitor at the L5 point [e.g., Vourlidas, 2015], on the same $1 \mathrm{AU}$ orbit as Earth, but lagging Earth with an azimuthal offset of $60^{\circ}$ (i.e., a lead time of $\sim 4.5$ days). 


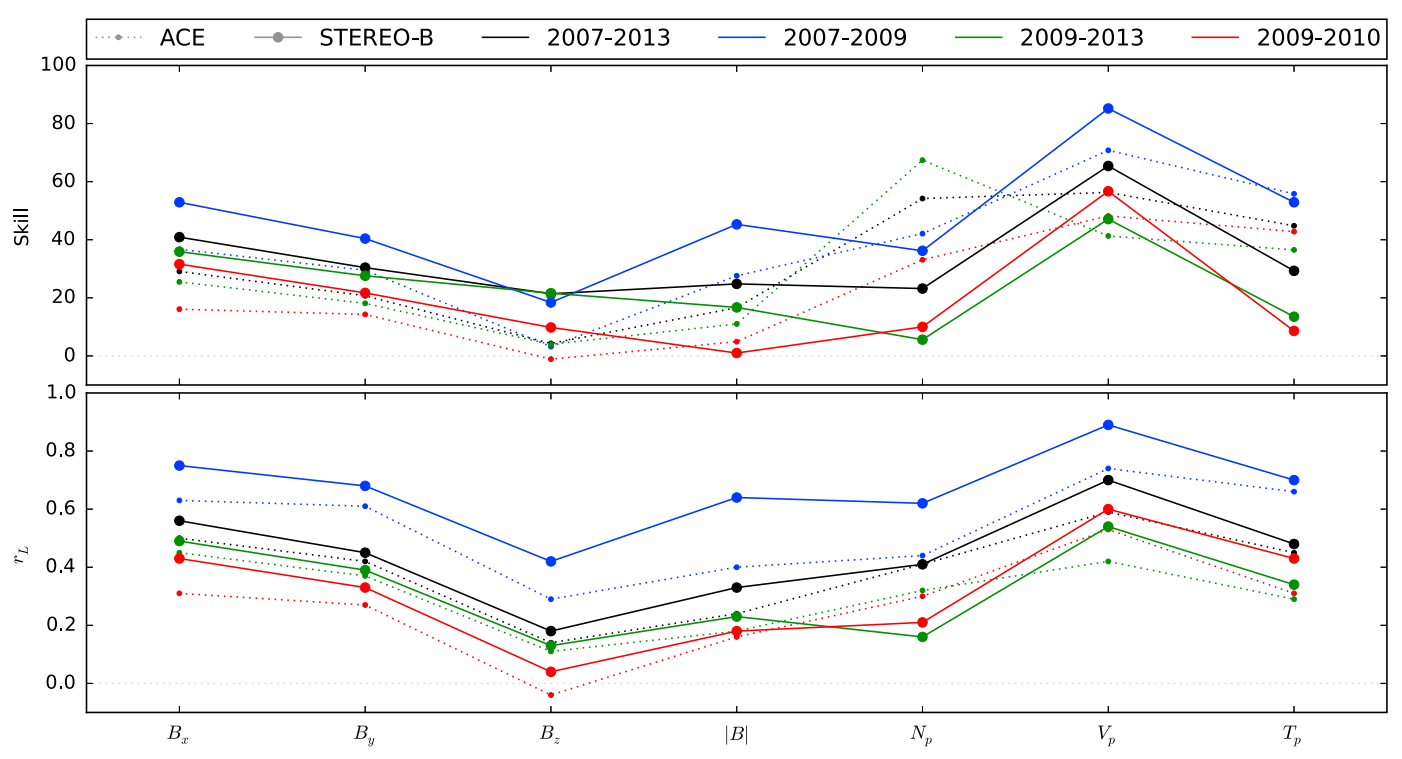

Figure 7. (top) Skill scores and (bottom) correlation coefficients for ACE (dashed lines) and STEREO-B (solid lines) evaluated over different periods. Years 2007-2013 (black line) show the entire period, corresponding to the results in Table 2, 2007-2009 (blue) show the period when the STEREO-B azimuthal angle $\Theta$ was $<50^{\circ}, 2009-2013$ (green) the period when $\Theta$ was $>50^{\circ}$, and $2009-2010$ (red) the 6 month period (2 August 2009 to 29 January 2010) when $50^{\circ}<\Theta<70^{\circ}$, i.e., the period roughly corresponding to the $\Theta=60^{\circ}$ values of an $\mathrm{L} 5$ mission.

Figure 7 examines this issue, showing skill scores and correlation coefficients for ACE and STEREO-B over different periods, for all the solar wind parameters in the persistence forecast. The black lines show the results for the entire 2007-2013 period and correspond to the results in Table 2. Blue lines show the results from 2007 to 2009, when the STEREO-B azimuthal angle $\Theta$ was $<50^{\circ}$, while green lines show results from 2009 to 2013 , when $\Theta$ was $>50^{\circ}$. Red lines show the $\sim 6$ month period from 2 August 2009 to 29 January 2010 when $\Theta$ was between $50^{\circ}$ and $70^{\circ}$, i.e., the period roughly corresponding to the $60^{\circ}$ azimuthal angle which an L5 mission would have.

Some interesting conclusions can be drawn from Figure 7, regarding data intercompatibility, the interpretation of STEREO-B persistence forecast results for L5 purposes, and implications for L5 particle instruments. These are elaborated on below.

\subsection{Data Intercompatibility}

Consistent with Figure 4, it is seen that the principal contribution to the typically better correlation and skill from STEREO-B (compared to ACE) in 2007-2013 comes from the 2007-2009 period, where the relative performance of STEREO-B over ACE is higher than in 2009-2013. It is notable that the exceptions to this better STEREO-B performance occur for the higher plasma moments (skill for $T_{p}$, both measures for $N_{p}$ ) and are more pronounced in the later 2009-2013 period. One possibility for this, consistent with the previously discussed (section 3) hypothesis of intercalibration between ACE and STEREO-B, may be if degradation of the plasma instruments on STEREO-B is gradually deteriorating the relative calibration of the higher plasma moments relative to ACE. (An alternative explanation might be a more rapid degradation of the ACE plasma instruments.)

Further investigation is needed to determine if intercalibration between ACE and STEREO-B is indeed responsible for the typically worse performance of STEREO-B for $T_{p}$ and $N_{p}$. If this hypothesis proves correct, it demonstrates that although a priority for operational space weather spacecraft must remain near-real-time delivery of data (which will thus not even benefit from the processing already performed on the science-level data used here), it is also necessary to ensure data from different operational spacecraft (such as missions to L1 and L5) are as compatible as possible. Specifically, in order to allow effective development of applications such as the persistence forecasts investigated here, best efforts must be made to ensure their instruments (or at least data) are similar, intercalibrated, and corrected for any gradual deterioration effects present. 


\subsection{Persistence Forecast Skill: Care Needed Using STEREO-B as a Proxy for L5}

The 2009-2010 results show that individually, for both ACE and STEREO-B, the absolute performance of the persistence forecast is typically worse than in all the other periods. The most likely explanation for this worse performance is that the solar cycle effect is getting conflated with the longitude-separation effect: this short period lies almost entirely during 2009, when there were very few recurrent high-speed streams compared to the periods before and after [Toma, 2010] and hence a reduction in a significant contributor to the fundamental mechanism responsible for skill in the persistence forecast. Note that this reduction in performance is hence merely a consequence of the fact that STEREO-B happened to pass through the $\Theta \sim 60^{\circ}$ location during this reduction in high-speed streams - a L5 mission present over longer periods at $\Theta=60^{\circ}$ would hence not be expected to suffer such a reduction in absolute performance.

Despite this absence of high-speed streams, it is seen that during 2009-2010, the STEREO-B results typically show an improvement (other than for higher-order plasma moments) over ACE, with relative improvements broadly consistent with other periods. The exception to this is skill for $|B|$, where STEREO-B results are slightly worse than $\mathrm{ACE}$ - this may be due to the reduction in high-speed streams during this period increasing the relative importance of heliolatitudinal separation - as seen in Figure 3, STEREO-B heliolatitudinal separations from ACE are biased toward negative values over this 6 month period (ranging from approximately $3^{\circ}$ to $-10^{\circ}$ ).

Consequently, the overall results suggest that despite the unfortunate conflating influence of reduced high-speed streams, persistence forecasts from STEREO-B during the $\Theta \sim 60^{\circ}$ period typically still show a relative improvement over $A C E$, and consequently, a mission to $L 5$ is also likely to result in an improvement to persistence forecasts. This is likely to be of lesser impact than the other advantages of such a mission, such as heliographic imaging of the Sun-Earth line for CME forecasting purposes [Vourlidas, 2015] but can be viewed as a side benefit. It is important to note also that the highest absolute performance of STEREO-B during the 2007-2009 period when the azimuthal angle $\Theta$ was $<50^{\circ}$ should not be viewed as an argument, based on the results here, for a mission closer to Earth - note that this better performance would come at the expense of a reduction in forecast lead time (less than the $\sim 4.5$ days lead time given by a mission at L5), and so such an argument would require careful analysis of the relative benefits of accuracy and lead time, not considered here.

\subsection{L5 Particle Instrumentation}

Finally, it is also interesting to consider the implications for an L5 mission of section 2, where it was seen that an increase in persistence forecast skill could be achieved by using compositional information to distinguish transient events (such as ICMEs), and hence to remove them from the persistence forecast, replacing the contaminated section with uncontaminated section from a prior rotation (the consequent price being a reduced correlation [Owens et al., 2013], due to an increased requirement on coronal stability). This demonstrates the advantages of including in situ solar wind monitors in the payload of a mission to L5. As discussed in section 2.2, the best performance (from an individual indicator) seen in Table 1 came from using the iron charge state as the transient filter and removing sections of $24 \mathrm{~h}$ either side of regions identified as being contaminated.

In principle, this could be used as an argument for including instruments capable of making plasma composition measurements on a mission to $L 5$, in addition to instruments for other in situ measurements of bulk plasma properties and magnetic field. However, it is important to consider the overall payload requirements for such a mission and the relative benefits from each instrument. Given the likely lesser impact of better persistence forecasts (compared, say, to operational heliographic imaging of the Sun-Earth line for identification of Earth-bound CMEs), one can also look at the Table 1 results and consider that the transient filtering using helium abundance and proton temperature also showed improvements relative to the standard persistence forecast. Although associated average skills are always slightly worse than equivalents using heavy ions, these light-ion-based measurements still show improvements for all individual parameters (except velocity, as discussed in section 2.2). More importantly, the necessary proton temperature or helium abundance measurements may well be capable of being made by a bulk plasma instrument on a L5 mission (if it is similar to SWEPAM), rather than requiring an equivalent to SWICS. Note that although on average, the proton temperature indicator always performs worse than the equivalent helium-based equivalent, it does not necessarily follow that a L5-based mission would be able to use a helium-based indicator for persistence forecasting, unless the L5 mission makes helium measurements available in near real time. Relying on a proton 
temperature-based indicator for L5 will also be compatible with our current aims for DSCOVR data (including efforts to increase skill by optimizing the $T_{\mathrm{ex}} / T_{p}$ threshold), as discussed in section 4.

\section{Conclusions}

In this paper, we have shown that a traditional 27 day persistence forecast model can be improved by applying techniques to remove CMEs from the observations used to create the forecast. The best individual indicator of CME presence was found to be the mean iron charge state $\left\langle Q_{\mathrm{Fe}}\right\rangle$, with the best performance (averaged across all solar parameters) achieved when $24 \mathrm{~h}$ intervals in either side of indicator triggering are removed and replaced by solar wind from a previous rotation. This is the preferred indicator, as it yielded very similar average performance to the (marginally better) results achieved using a more complex combination of all indicators (proton temperature, Fe and O charge state, and He abundance). The result of the Fe-based CME removal is an improvement for skill scores across all solar wind parameters; in particular, the skill score for the southward IMF component $B_{z}$, a crucial parameter for determining solar wind geoeffectiveness, went from $3.5 \%$ to $19.3 \%$, a 15.8 percentage point improvement. The equivalent skill score for an alternative indicator, the proton temperature, also shows a 7.7 percentage point improvement in $B_{z}$ (to $11.2 \%$ ) - this is lower but should be more representative of improvements which may be expected operationally from DSCOVR data (given heavy ion indicators will not be available from DSCOVR).

We have also shown that a reduced lead time persistence forecast can be built, using STEREO-A/B data to ease the requirement of persistence forecasts from spacecraft at the L1 Lagrangian point, namely, that coronal solar wind sources must remain unchanged for multiples of a whole synodic period ( $\sim 27$ days). Using STEREO data reduces the forecast lead time but allows the forecast to be based on more recently observed data. This results in an improvement in the skill scores of many solar wind parameters $-B_{z}$ skill scores improved from $4.3 \%$ to $21.4 \%$. Exceptions to this improvement are seen for the proton density and the proton temperature, where the STEREO-based skill scores worsen - we suggest the most likely cause for this may be due to calibration differences between instruments on the ACE and STEREO spacecraft. Correlation coefficients, which should be less sensitive to calibration issues, show improvement for all solar wind parameters. The impact of the heliolatitudinal difference between STEREO and ACE (ranging between $\sim \pm 14^{\circ}$, meaning the spacecraft may observe different solar wind streams) was investigated by comparing the power spectrum of the heliolatitudinal differences and the correlation between the ACE and time-shifted STEREO observations; only limited impact was found, principally on $B_{z}$. No investigation has been done into the effects of the orbit radius differences of ACE and STEREO — this may have some effect on timing (R. Bentley, personal communication, 2014), but we expect this to be minor, less important than the previously mentioned heliolatitudinal effects. Further work is needed to verify this.

We have investigated the $\sim 6$ month period when STEREO-B was in a position near the Lagrangian L5 point (in the context of the longer periods before and after), aiming to determine what impact a future L5 mission may have on a reduced lead time forecast (with lead times of $\sim 4.5$ days). We have found that the STEREO-B-based persistence forecast still typically shows better performance than the equivalent ACE-based results, implying that improved L5-based persistence forecasts (relative to L1-based persistence forecasts) will be a minor benefit of a L5 mission. We note though that the absolute performance of the persistence forecast (for both spacecraft) is worse than in other periods, likely due to the relative absence of recurrent high-speed streams during this period, hence a reduction in a significant contributor to the fundamental mechanism responsible for skill in the persistence forecast. We also note a $L 5$ mission may be able to filter CMEs using a proton temperature-based indicator (compatible with DSCOVR) and that effective persistence forecasts will require efforts to ensure $L 1$ and $L 5$ data are intercompatible.

The regular persistence model and the reduced lead time persistence models were transitioned to operations at the Met Office in the spring of 2015 and are now routinely used by forecasters, as a complement to dynamical models, which are known to underperform compared to persistence models when looking at high-speed enhancements [Owens et al., 2013]. Initial feedback from forecasters seems to agree with these findings but a thorough event-based validation will be discussed in a follow-up paper. Due to the SWICS instrument onboard ACE suffering some hardware damage in August 2011, the heavy ion measurement methodology was altered. The impact of using the post 2011 data set will be investigated shortly, but in the meantime, CME-filtering is not being performed. Future work will also need to be performed when DSCOVR replaces $A C E$, to adapt CME-filtering to the available CME indicators from the PlasMag instrument, and to implement routine assessment of the persistence forecast skill score. 


\section{Acknowledgments}

We thank the referees for helpful feedback, which has helped improve this paper. We gratefully acknowledge useful discussions with Rosemary Challen and Alice Burton (née Wardle), whose Masters work provided important input to this research. P.K. would like to acknowledge the support of a UK STFC PhD studentship. The work presented here was done principally via the Met Office summer placement programme. AGU data policy: the open data sources used have been provided in text; code can be obtained from the corresponding author on request.

\section{References}

Acuña, M. H., D. Curtis, J. L. Scheifele, C. T. Russell, P. Schroeder, A. Szabo, and J. G. Luhmann (2007), The STEREO/IMPACT magnetic field experiment, Space Sci. Rev., 136(1-4), 203-226, doi:10.1007/s11214-007-9259-2.

Bolduc, L. (2002), GIC observations and studies in the Hydro-Qubec power system, J. Atmos. Sol. Terr. Phys., 64(16), 1793-1802, doi:10.1016/S1364-6826(02)00128-1.

Burlaga, L., E. Sittler, F. Mariani, and R. Schwenn (1981), Magnetic loop behind an interplanetary shock: Voyager, Helios, and IMP 8 observations, J. Geophys. Res., 86(A8), 6673-6684, doi:10.1029/JA086iA08p06673.

Burlaga, L. F., and R. P. Lepping (1977), The causes of recurrent geomagnetic storms, Planet. Space Sci., 25(12), 1151-1160, doi:10.1016/0032-0633(77)90090-3.

Cane, H. V., and I. G. Richardson (2003), Interplanetary coronal mass ejections in the near-Earth solar wind during 1996-2002, J. Geophys. Res., 108(A4), 1156, doi:10.1029/2002JA009817.

Cannon, P. S. (2013), Extreme Space Weather -A report published by the UK Royal Academy of Engineering, Space Weather, 11(4), 138-139, doi:10.1002/swe.20032.

Carrington, R. C. (1859), Description of a singular appearance seen in the Sun on September 1, 1859, Mon. N. R. Astron. Soc., 20(1), 13-15, doi:10.1093/mnras/20.1.13

Dyer, C. (2002), Radiation effects on spacecraft \& aircraft, in Proceedings of the 2nd Solar Cycle and Space Weather Euroconference, SOLSPA 2001, vol. 477, edited by H. Sawaya-Lacoste, pp. 505-512, ESA Special Publ., Vico Equense, Italy.

Galvin, A. B., et al. (2008), The Plasma and Suprathermal Ion Composition (PLASTIC) investigation on the STEREO observatories, Space Sci. Rev., 136(1-4), 437-486, doi:10.1007/s11214-007-9296-x.

Geiss, J., G. Gloeckler, and R. V. Steiger (1995), Origin of the solar wind from composition data, Space Sci. Rev., 72(1-2), 49-60, doi:10.1007/BF00768753.

Gibbs, M., and M. M. Bisi (2015), Space weather-The economic case, Abstract SH21B-2398 presented at the 2015 Fall Meeting, AGU, San Francisco, Calif. 14-18 Dec.

Gloeckler, G., et al. (1998), Investigation of the composition of solar and interstellar matter using solar wind and pickup ion measurements with SWICS and SWIMS on the ACE spacecraft, Space Sci. Rev., 86(1-4), 497-539, doi:10.1023/A:1005036131689.

Gosling, J. T. (1990), Coronal mass ejections and magnetic flux ropes in interplanetary space, in Physics of Magnetic Flux Ropes, edited by J. T. Gosling, pp. 343-364, AGU, Washington, D. C

Gosling, J. T., V. Pizzo, and S. J. Bame (1973), Anomalously low proton temperatures in the solar wind following interplanetary shock waves evidence for magnetic bottles?, J. Geophys. Res., 78(13), 2001 -2009, doi:10.1029/JA078i013p02001.

Gosling, J. T., D. N. Baker, S. J. Bame, W. C. Feldman, R. D. Zwickl, and E. J. Smith (1987), Bidirectional solar wind electron heat flux events, J. Geophys. Res., 92(A8), 8519-8535, doi:10.1029/JA092iA08p08519.

Gosling, J. T., D. J. McComas, J. L. Phillips, and S. J. Bame (1991), Geomagnetic activity associated with Earth passage of interplanetary shock disturbances and coronal mass ejections, J. Geophys. Res., 96(A5), 7831-7839, doi:10.1029/91JA00316.

Hapgood, M., and A. Thomson (2010), Space weather: Its impact on Earth and implications for business.

Hirshberg, J., S. J. Bame, and D. E. Robbins (1972), Solar flares and solar wind helium enrichments: July 1965-1967, Sol. Phys., 23(2), 467-486, doi:10.1007/BF00148109.

Jonas, S., and E. D. McCarron (2016), White house releases national space weather strategy and action plan, Space Weather, 14(2), 54-55, doi:10.1002/2015SW001357.

Kaiser, M. L. (2005), The STEREO mission: An overview, Adv. Space Res., 36(8), 1483-1488, doi:10.1016/j.asr.2004.12.066.

Kilchenmann, A. (2007), Interplanetary coronal mass ejections observed with SWICS/Ulysses, PhD thesis, Univ. of Bern, Switzerland.

Lepri, S. T., and T. H. Zurbuchen (2004), Iron charge state distributions as an indicator of hot ICMEs: Possible sources and temporal and spatial variations during solar maximum, J. Geophys. Res., 109, A01112, doi:10.1029/2003JA009954.

Lepri, S. T., T. H. Zurbuchen, L. A. Fisk, I. G. Richardson, H. V. Cane, and G. Gloeckler (2001), Iron charge distribution as an identifier of interplanetary coronal mass ejections, J. Geophys. Res., 106(A12), 29,231-29,238, doi:10.1029/2001JA000014.

Lopez, R. E. (1987), Solar cycle invariance in solar wind proton temperature relationships, J. Geophys. Res., 92(A10), 11,189-11,194, doi:10.1029/JA092iA10p11189.

Manoharan, P. K. (2006), Evolution of coronal mass ejections in the inner heliosphere: A study using white-light and scintillation images, Sol. Phys., 235(1-2), 345-368, doi:10.1007/s11207-006-0100-y.

McComas, D. J., S. J. Bame, P. Barker, W. C. Feldman, J. L. Phillips, P. Riley, and J. W. Griffee (1998), Solar Wind Wlectron Proton Alpha Monitor (SWEPAM) for the advanced composition explorer, Space Sci. Rev., 86(1-4), 563-612, doi:10.1023/A:1005040232597.

McComas, D. J., H. A. Elliott, N. A. Schwadron, J. T. Gosling, R. M. Skoug, and B. E. Goldstein (2003), The three-dimensional solar wind around solar maximum, Geophys. Res. Lett., 30(10), 1517, doi:10.1029/2003GL017136.

Montgomery, M. D., J. R. Asbridge, S. J. Bame, and W. C. Feldman (1974), Solar wind electron temperature depressions following some interplanetary shock waves: Evidence for magnetic merging?, J. Geophys. Res., 79(22), 3103-3110, doi:10.1029/JA079i022p03103.

Neugebauer, M., and R. Goldstein (1997), Particle and field signatures of coronal mass ejections in the solar wind, in Coronal Mass Ejections, pp. 245-251, AGU, Washington, D. C., doi:10.1029/GM099p0245.

Owens, M. J., R. Challen, J. Methven, E. Henley, and D. R. Jackson (2013), A 27 day persistence model of near-Earth solar wind conditions: A long lead-time forecast and a benchmark for dynamical models, Space Weather, 11(5), 225-236, doi:10.1002/swe.20040.

Paschmann, G., and P. W. Daly (1998), Analysis Methods for Multi-Spacecraft Data, ISSI Scientific Reports Series SR-001, vol. 1, ESA/ISSI.

Reinard, A. A., T. H. Zurbuchen, L. A. Fisk, S. T. Lepri, R. M. Skoug, and G. Gloeckler (2001), Comparison between average charge states and abundances of ions in CMEs and the slow solar wind, in AIP Conference Proceedings, vol. 598, pp. 139-144, AIP Publ., doi:10.1063/1.1433992

Richardson, I. G. (2013), The formation of CIRs at stream-stream interfaces and resultant geomagnetic activity, in Recurrent Magnetic Storms: Corotating Solar Wind Streams, pp. 45-58, AGU, Washington, D. C., doi:10.1029/167GM06

Richardson, I. G., and H. V. Cane (1995), Regions of abnormally low proton temperature in the solar wind (1965-1991) and their association with ejecta, J. Geophys. Res., 100(A12), 23,397-23,412, doi:10.1029/95JA02684.

Richardson, I. G., and H. V. Cane (2004), Identification of interplanetary coronal mass ejections at 1 AU using multiple solar wind plasma composition anomalies, J. Geophys. Res., 109(A9), A09104, doi:10.1029/2004JA010598.

Richardson, I. G., and H. V. Cane (2010), Near-earth interplanetary coronal mass ejections during solar cycle 23 (1996-2009): Catalog and summary of properties, Sol. Phys., 264(1), 189-237, doi:10.1007/s11207-010-9568-6.

Samuelson, H. (2015), DSCOVR_PlasMag Instrument Info Sheet. [Available at http://www.nesdis.noaa.gov/DSCOVR/ spacecraft.html05-Aug-2016.] 
Schrijver, C. J., R. Dobbins, W. Murtagh, and S. M. Petrinec (2014), Assessing the impact of space weather on the electric power grid based on insurance claims for industrial electrical equipment, Space Weather, 12(7), 487-498, doi:10.1002/2014SW001066.

Schrijver, C. J., et al. (2015), Understanding space weather to shield society: A global road map for 2015 to 2025 commissioned by COSPAR and ILWS, Adv. Space Res., 55(12), 2745-2807, doi:10.1016/j.asr.2015.03.023.

Shearer, P., J. M. Raines, S. T. Lepri, J. A. Gilbert, M. J. Weberg, G. Gloeckler, and T. H. Zurbuchen (2014), SWICS 2.0 level 3 version 1.11 data release notes, [Online; accessed 30-June-2015].

Smith, C. W., J. L'Heureux, N. F. Ness, M. H. Acua, L. F. Burlaga, and J. Scheifele (1998), The ACE magnetic fields experiment, Space Sci. Rev., 86(1-4), 613-632, doi:10.1023/A:1005092216668.

Spence, H., D. Baker, A. Burns, T. Guild, C.-L. Huang, G. Siscoe, and R. Weigel (2004), Center for integrated space weather modeling metrics plan and initial model validation results, J. Atmos. Sol. Terr. Phys., 66(15-16), 1499-1507, doi:10.1016/j.jastp.2004.03.029.

Sreeja, V., M. Aquino, K. de Jong, and H. Visser (2014), Effect of the 24 September 2011 solar radio burst on precise point positioning service, Space Weather, 12(3), 143-147, doi:10.1002/2013SW001011.

Stewart, B. (1861), On the great magnetic disturbance which extended from August 28 to September 7, 1859, as recorded by photography at the Kew observatory, Philos. Trans. R. Soc. London, 151, 423-430, doi:10.1098/rstl.1861.0023.

Stone, E. C., A. M. Frandsen, R. A. Mewaldt, E. R. Christian, D. Margolies, J. F. Ormes, and F. Snow (1998), The advanced composition explorer, Space Sci. Rev., 86(1-4), 1-22, doi:10.1023/A:1005082526237.

Toma, G. (2010), Evolution of coronal holes and implications for high-speed solar wind during the minimum between cycles 23 and 24 , Sol. Phys., 274(1), 195-217, doi:10.1007/s11207-010-9677-2.

Vourlidas, A. (2015), Mission to the Sun-Earth 15 Lagrangian point: An optimal platform for space weather research, Space Weather, 13(4), 197-201, doi:10.1002/2015SW001173.

Vršnak, B., D. Vrbanec, and J. Čalogović (2008), Dynamics of coronal mass ejections, $A$ \& A, 490(2), 811-815, doi:10.1051/0004-6361:200810215.

Wardle, A. (2013), Improving a persistence forecast of the near-Earth solar wind, Master's thesis, Univ. of Reading.

Webb, D. F., E. W. Cliver, N. U. Crooker, O. C. St. Cyr, and B. J. Thompson (2000), Relationship of halo coronal mass ejections, magnetic clouds, and magnetic storms, J. Geophys. Res., 105(A4), 7491-7508, doi:10.1029/1999JA000275.

Wicks, R. T., M. J. Owens, and T. S. Horbury (2010), The variation of solar wind correlation lengths over three solar cycles, Sol. Phys., 262(1), $191-198$, doi:10.1007/s11207-010-9509-4.

Yashiro, S., N. Gopalswamy, G. Michalek, O. C. St. Cyr, S. P. Plunkett, N. B. Rich, and R. A. Howard (2004), A catalog of white light coronal mass ejections observed by the SOHO spacecraft, J. Geophys. Res., 109(A7), A07105, doi:10.1029/2003JA010282.

Yermolaev, Y. I., and V. V. Stupin (1997), Helium abundance and dynamics in different types of solar wind streams: The Prognoz 7 observations, J. Geophys. Res., 102(A2), 2125-2136, doi:10.1029/96JA01635.

Zurbuchen, T. H., and I. G. Richardson (2006), In-situ solar wind and magnetic field signatures of interplanetary coronal mass ejections, in Coronal Mass Ejections, vol. 21 in Space Sciences Series of ISSI, pp. 31-43, Springer, New York. 